\title{
Charm Physics at BESIII
}

\author{
Lei Li (For BESIII Collaboration) ${ }^{* i t}$ \\ Beijing Institute of Petro-chemical Technology \\ E-mail: lilei@ihep.ac.cn
}

Based on 2.93 and $0.567 \mathrm{fb}^{-1}$ data taken at the center-of-mass energies $\sqrt{s}=3.773$ and 4.599 $\mathrm{GeV}$ with the BESIII detector at the BEPCII collider, we report the precise measurements of the decay constant $f_{D^{+}}$, the form factors of $D$ semileptonic decays, the Dalitz plot analysis of $D^{+} \rightarrow K_{S}^{0} \pi^{+} \pi^{0}$, the strong phase measurement in $D^{0} \rightarrow K^{-} \pi^{+}$, the $D^{0} \bar{D}^{0}$ mixing parameter $y_{\mathrm{CP}}$, the observation of the singly-cabibbo-suppressed hadronic decays $D^{+} \rightarrow \omega \pi^{+}$and $D^{0} \rightarrow \omega \pi^{0}$, as well as the first absolute measurements of the branching fractions for $\Lambda_{c}^{+} \rightarrow \Lambda e^{+} v_{e}, \Lambda_{c}^{+}$decays to 12 hadronic final states and observation of $\Lambda_{c}^{+} \rightarrow n K_{S}^{0} \pi^{+}$.

54th International Winter Meeting on Nuclear Physics

25-29 January 2016

Bormio, Italy

\footnotetext{
* Speaker.

${ }^{\dagger}$ I would like to thank for the support of the National Natural Science Foundation of China (NSFC) under Contracts No. 11505010, and Beijing municipal government under Contract Nos. KM201610017009, 2015000020124G064.
} 


\section{Introduction}

Precision measurements of charm decays provide rich information to better understand strong and weak effects. Firstly, the deferential decay rates of the $D$ leptonic and semileptonic decays can be simply functioned as decay constant $f_{D^{+}}$or form factors and CKM matrix element $\left|V_{c s(d)}\right|$. From analysis of the $D$ leptonic and semileptonic decays, we can determine these elementary constants, thus calibrating the LQCD calculation on $f_{D^{+}}$and the form factors and testing the CKM matrix unitarity.

Secondly, studies of $D$ hadronic decays are important due to several aspects. At $\psi(3770)$ resonance peak, the quantum correction property of $D^{0}$ meson production provides an access to $\mathrm{CP}$ asymmetry in $D^{0} \bar{D}^{0}$ mixing and strong phase parameters which can be used to constrain $\gamma / \phi_{3}$ and to further test the CKM matrix unitarity. Improved knowledge of singly-cabibbo-suppressed (SCS) decays is helpful for understanding of $U$-spin and $S U(3)$ flavor symmetry breaking effects. Datlitz plot analysis of three-body decays can provide rich information about the parameters of sub-resonances and strong phases.

Thirdly, in the Standard Model (SM), the Flavor Changed Neutral Current (FCNC) process and the Leptonic Number Violation (LNV) process are highly suppressed. However, some new dynamics beyond the SM may enhance these kinds of processes to observable level at BESIII. So, search for these rare decays can be used to probe for new physics beyond the SM. Any evidence of rare decay and CP violation in charm decays or significant deviation of CKM unitarity may indicate new physics beyond the SM.

Finally, compared to charmed meson decays, the knowledge of charmed baryon $\Lambda_{c}^{+}$decays is still very poor. Currently, the total measured branching fractions for $\Lambda_{c}^{+}$is still not more than $65 \%$ and lots of the decay modes are unknown [1]. Thus, it is desired to improve the measurements of the known decays and search for new decay modes. Significantly improved knowledge of the decay rates or dynamics of charm decays can also provide better inputs for beauty physics.

We report recent results on the studies of the leptonic, semleptonic and hadronic decays of $D^{0}$, $D^{+}$and $\Lambda_{c}^{+}$. These are based on 2.93 [2] and 0.567 [3] $\mathrm{fb}^{-1}$ data at $\sqrt{s}=3.773$ and $4.599 \mathrm{GeV}$ collected with the BESIII detector [4]. The charge conjugation is always implied.

\section{2. $D$ leptonic and semileptonic decay}

In the Standard Model, the $D^{+}$mesons decay into $\ell v_{\ell}$ via a virtual $W^{+}$boson. The decay rate of the leptonic decays $D^{+} \rightarrow \ell^{+} v_{\ell}$ can be parameterized by the $D^{+}$decay constant $f_{D^{+}}$via

$$
\Gamma\left(D^{+} \rightarrow \ell^{+} v_{\ell}\right)=\frac{G_{F}^{2}}{8 \pi}\left|V_{c d}\right|^{2} f_{D^{+}}^{2} m_{\ell}^{2} m_{D^{+}}\left(1-\frac{m_{\ell}^{2}}{m_{D^{+}}^{2}}\right)
$$

where $G_{F}$ is the Fermi coupling constant, $\left|V_{c d}\right|$ is the quark mixing matrix element, $m_{\ell}$ and $m_{D^{+}}$ are the lepton and $D^{+}$masses. To investigate the leptonic decay $D^{+} \rightarrow \mu^{+} v_{\mu}$ [5], the singly tagged $D^{-}$mesons are reconstructed using 9 hadronic decays $K^{+} \pi^{-} \pi^{-}, K_{S}^{0} \pi^{-}, K_{S}^{0} K^{-}, K^{+} K^{-} \pi^{-}$, $K^{+} \pi^{-} \pi^{-} \pi^{0}, \pi^{+} \pi^{-} \pi^{-}, K_{S}^{0} \pi^{-} \pi^{0}, K^{+} \pi^{+} \pi^{-} \pi^{-} \pi^{-}$and $K_{S}^{0} \pi^{+} \pi^{-} \pi^{-}$. From these, we accumulate $(170.31 \pm 0.34) \times 10^{4}$ singly tagged $D^{-}$mesons. Fig. 1 shows the $M_{\text {miss }}^{2}$ distribution of the 


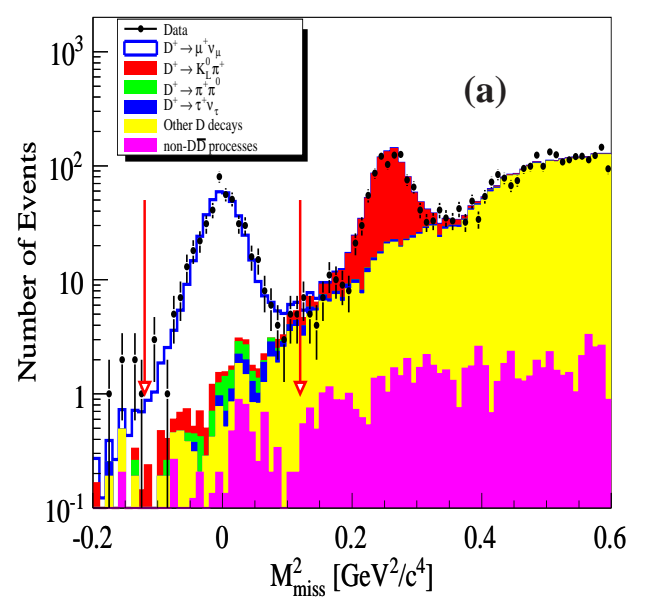

Figure 1: The $M_{\text {miss }}^{2}$ distribution of $D^{+} \rightarrow \mu^{+} v_{\mu}$.

$D^{+} \rightarrow \mu^{+} v_{\mu}$ candidates, which are selected in the systems against the singly tagged $D^{-}$mesons. We obtain $409 \pm 21$ net $D^{+} \rightarrow \mu^{+} v_{\mu}$ signals and measured the branching fraction $\mathscr{B}\left(D^{+} \rightarrow\right.$ $\left.\mu^{+} v_{\mu}\right)=\left(3.71 \pm 0.19_{\text {stat. }} \pm 0.06_{\text {sys. }}\right) \times 10^{-4}$. Using the measured $\mathscr{B}\left(D^{+} \rightarrow \mu^{+} v_{\mu}\right)$ and the quark mixing matrix element $\left|V_{c d}\right|$ from a global Standard Model fit [1], we determine the $D^{+}$decay constant $f_{D^{+}}=203.2 \pm 5.3_{\text {stat. }} \pm 1.8_{\text {sys. }} \mathrm{MeV}$. The $\mathscr{B}\left(D^{+} \rightarrow \mu^{+} v_{\mu}\right)$ and $f_{D^{+}}$measured at BESIII are consistent within errors with previous measurements, but with the best precision. By using the measured $B\left(D^{+} \rightarrow \mu^{+} v_{\mu}\right)$ and the LQCD calculation on $f_{D^{+}}$[6], we determine $\left|V_{c d}\right|=$ $0.2210 \pm 0.058_{\text {stat. }} \pm 0.047_{\text {sys. }}$, which has the best precision in the world to date.

On the other hand, the $D$ semileptonic decays can be parameterized by the quark mixing matrix element and the form factor of hadronic weak current simply, thus providing an ideal window to probe for the weak and strong effects. For example, the differential decay rates of $D \rightarrow K(\pi) e^{+} v_{e}$ can be simply written as

$$
\frac{d \Gamma}{d q^{2}}=\frac{G_{F}^{2}}{24 \pi^{3}}\left|V_{c s(d)}\right|^{2} p_{K(\pi)}^{3}\left|f_{+}^{K(\pi)}\left(q^{2}\right)\right|^{2},
$$

where $G_{F}$ is the Fermi coupling constant, $\left|V_{c s(d)}\right|$ is the quark mixing matrix element, $p_{K(\pi)}$ is the kaon(pion) momentum in the $D^{0}$ rest frame, $f_{+}^{K(\pi)}\left(q^{2}\right)$ is the form factor of hadronic weak current depending on the square of the four momentum transfer $q=p_{D}-p_{K(\pi)}$. To investigate the semileptonic decays $D^{0} \rightarrow K(\pi)^{-} e^{+} v_{e}$ [7], we reconstruct the singly tagged $\bar{D}^{0}$ mesons using 5 hadronic decays of $K^{+} \pi^{-}, K^{+} \pi^{-} \pi^{0}, K^{+} \pi^{-} \pi^{-} \pi^{+}, K^{+} \pi^{-} \pi^{-} \pi^{+} \pi^{0}$ and $K^{+} \pi^{-} \pi^{0} \pi^{0}$, which give $(279.33 \pm 0.37) \times 10^{4}$ singly tagged $\bar{D}^{0}$ mesons. Base on $70727 \pm 278 D^{0} \rightarrow K^{-} e^{+} v_{e}$ and $6297 \pm 87$ and $D^{0} \rightarrow \pi^{-} e^{+} v_{e}$ signals, we determine the branching fractions $\mathscr{B}\left(D^{0} \rightarrow K^{-} e^{+} v_{e}\right)=$ $\left(3.505 \pm 0.014_{\text {stat. }} \pm 0.033_{\text {sys. }}\right) \%$ and $\mathscr{B}\left(D^{0} \rightarrow \pi^{-} e^{+} v_{e}\right)=\left(0.2950 \pm 0.0041_{\text {stat. }} \pm 0.0026_{\text {sys. }}\right) \%$, respectively. The branching fractions measured at BESIII are consistent within errors with previous measurements, but with the best precision. Fig. 2 shows the fits to the partial widths for $D^{0} \rightarrow K^{-} e^{+} v_{e}$ and $D^{0} \rightarrow \pi^{-} e^{+} v_{e}$ using the Simple Pole model [8], the Modified Pole model [8], the two-parameter series expansion (Series.2.Par.) [9] and the three-parameter series expansion (Series.3.Par.) [9]. From the fits, we obtain the parameters of different models. With the 

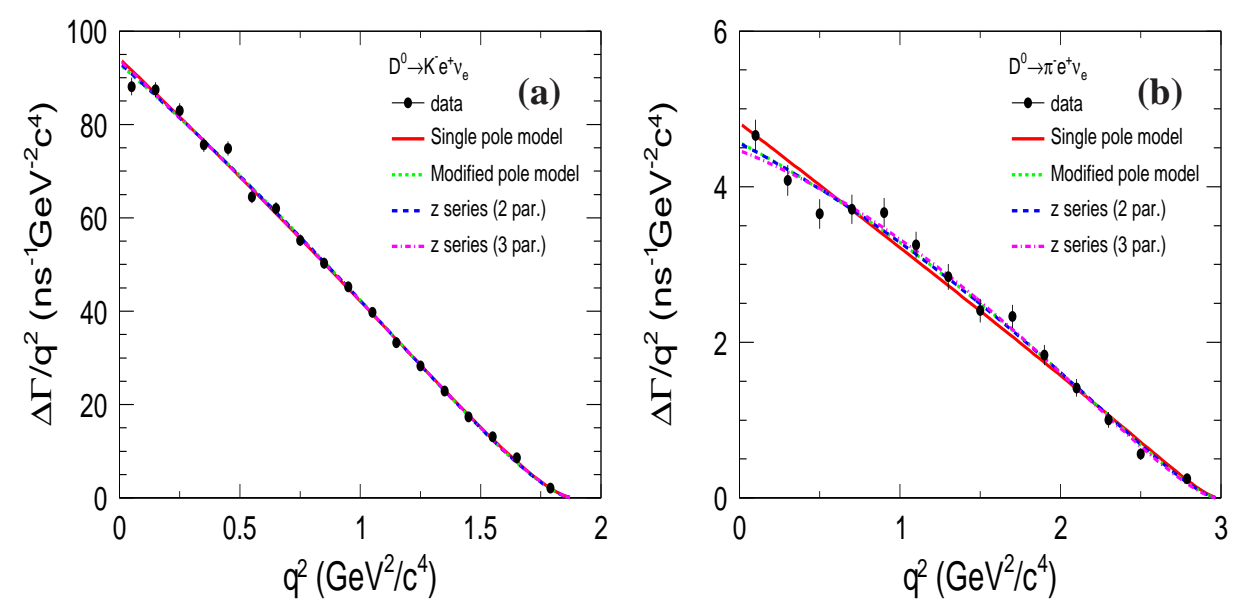

Figure 2: Fits to the partial widths of (a) $D^{0} \rightarrow K^{-} e^{+} v_{e}$ and (b) $D^{0} \rightarrow \pi^{-} e^{+} v_{e}$.

extracted $f_{+}^{K(\pi)}(0)\left|V_{c s(d)}\right|$ based on two-parameter series expansion and the expected $f_{+}^{K(\pi)}(0)$ by LQCD $[10,11]$, we determine the quark mixing matrix elements $\left|V_{c s(d)}\right|$.

To study the semileptonic decays $D^{+} \rightarrow K_{L}^{0} e^{+} v_{e}, D^{+} \rightarrow K^{-} \pi^{+} e^{+} v_{e}$ and $D^{+} \rightarrow \omega(\phi) e^{+} v_{e}$, we use 6 hadronic decays of $K^{+} \pi^{-} \pi^{-}, K^{+} \pi^{-} \pi^{-} \pi^{0}, K_{S}^{0} \pi^{-}, K_{S}^{0} \pi^{-} \pi^{0}, K_{S}^{0} \pi^{+} \pi^{-} \pi^{-}$and $K^{+} K^{-} \pi^{-}$. With about 24 thousands of $D^{+} \rightarrow K_{L}^{0} e^{+} v_{e}$ signals [12], we make first measurement of the branching fraction $\mathscr{B}\left(D^{+} \rightarrow K_{L}^{0} e^{+} v_{e}\right)=\left(4.482 \pm 0.027_{\text {stat. }} \pm 0.103_{\text {sys. }}\right) \%$ and the CP asymmetry $A_{\mathrm{CP}}^{D^{+} \rightarrow K_{L}^{0} e^{+} v_{e}}=$ $\left(-0.59 \pm 0.60_{\text {stat. }} \pm 1.50_{\text {sys. }}\right) \%$, supporting that there is no CP asymmetry in this decay. In addition, we perform simultaneous fit to the event density $I\left(q^{2}\right)$ for different tag modes with the twoparameter series expansion and obtain the product of $f_{+}^{K}(0)\left|V_{c s}\right|=0.728 \pm 0.006_{\text {stat. }} \pm 0.011_{\text {sys. }}$.

Using $18262 D^{+} \rightarrow K^{-} \pi^{+} e^{+} v_{e}$ candidates [13] which is almost background free, we determine the branching fraction $\mathscr{B}\left(D^{+} \rightarrow K^{-} \pi^{+} e^{+} v_{e}\right)=(3.71 \pm 0.03 \pm 0.08) \%$. A partial wave analysis (PWA) is performed on the selected candidates, with results shown in Fig. 3. The PWA results show that the dominant $\bar{K}^{* 0}$ component is accompanied by an $S$-wave contribution accounting for $(6.05 \pm 0.22 \pm 0.18) \%$ of the total rate, and other components can be negligible. We obtain the mass and width of $\bar{K}^{* 0}(892) M_{\bar{K}^{* 0}(892)}=(894.60 \pm 0.25 \pm 0.08) \mathrm{MeV} / c^{2}$ and $\Gamma_{\bar{K}^{* 0}(892)}=(46.42 \pm$ $0.56 \pm 0.15) \mathrm{MeV} / c^{2}$, the Blatt-Weisskopf parameter $r_{\mathrm{BW}}=3.07 \pm 0.26 \pm 0.11(\mathrm{GeV} / c)^{-1}$, as well as the parameters of the hadronic form factors $r_{V}=\frac{V(0)}{A_{1}(0)}=1.411 \pm 0.058 \pm 0.007, r_{2}=\frac{A_{2}(0)}{A_{1}(0)}=$ $0.788 \pm 0.042 \pm 0.008, m_{V}=\left(1.81_{-0.17}^{+0.25} \pm 0.02\right) \mathrm{MeV} / c^{2}, m_{A}=\left(2.61_{-0.17}^{+0.22} \pm 0.03\right) \mathrm{MeV} / c^{2}, A_{1}(0)=$ $0.585 \pm 0.011 \pm 0.017$. In the above PWA process, the phase of the non-resonant background $\delta_{S}\left(m_{K \pi}\right)$ is factorized by the LASS parameterizations, and the helicity form factors $H_{+}\left(q^{2}, m_{K \pi}\right)$, $H_{-}\left(q^{2}, m_{K \pi}\right)$ and $H_{0}\left(q^{2}, m_{K \pi}\right)$ are parameterized by the spectroscopic pole dominance (SPD) model [14]. We also make model-independent measurements of the $\delta_{S}\left(m_{K \pi}\right)$, and the helicity form factors, respectively. The results are consistent with the expectations of the corresponding models and previous measurements.

Based on $491 \pm 32 D^{+} \rightarrow \omega e^{+} v_{e}$ signals [15], we determine the branching fraction $\mathscr{B}\left(D^{+} \rightarrow\right.$ $\left.\omega e^{+} v_{e}\right)=\left(1.63 \pm 0.11_{\text {stat. }} \pm 0.08_{\text {sys. }}\right) \times 10^{-3}$, which is consistent with previous measurements but with better precision. We perform amplitude analysis of $D^{+} \rightarrow \omega e^{+} v_{e}$ for the first time, and obtain 

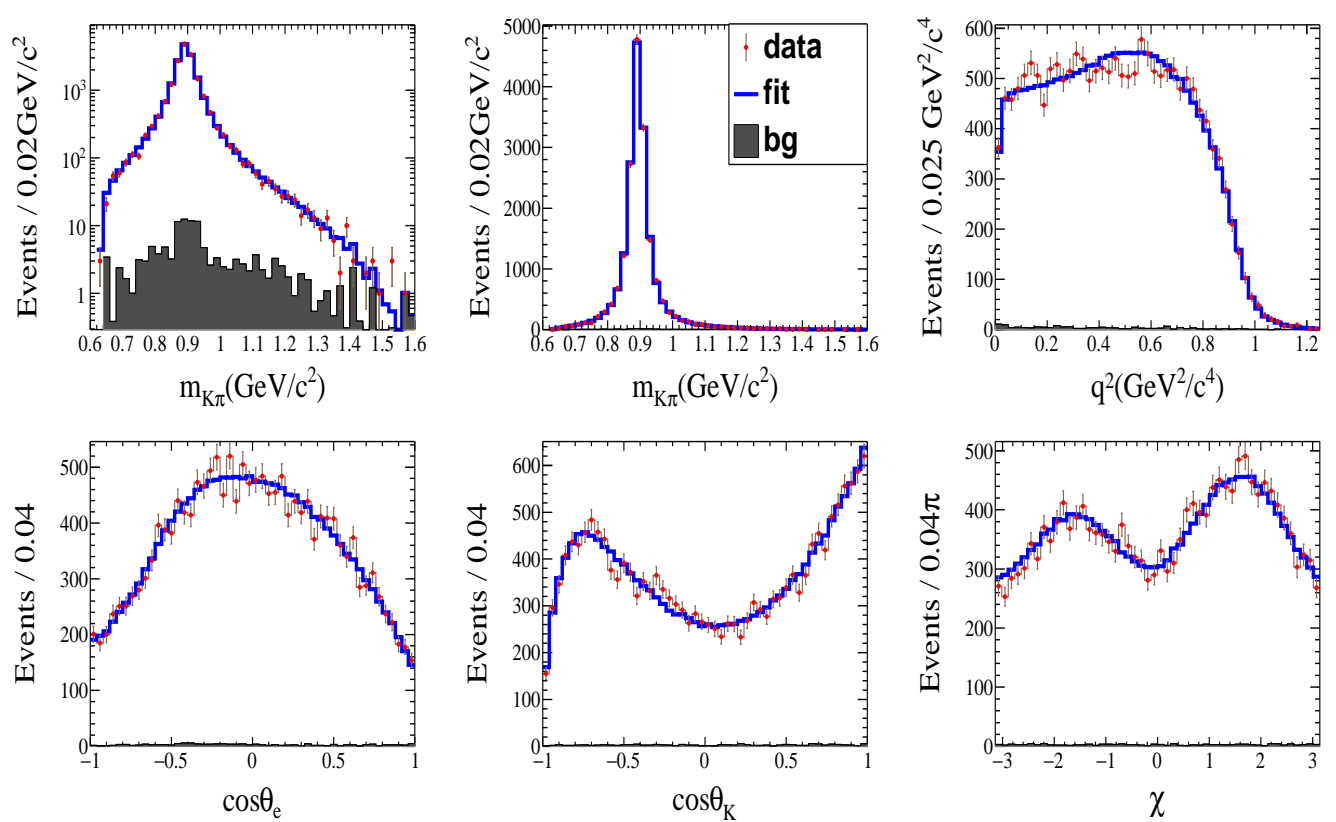

Figure 3: Projections of the kinematic variables of PWA for $D^{+} \rightarrow K^{-} \pi^{+} e^{+} v_{e}$, where $m_{K \pi}$ is the $K \pi$ mass, $q^{2}$ is the $e v_{e}$ mass square, $\theta_{K}$ is the angle between $\pi$ and $D$ momenta in the $K \pi$ rest frame, $\theta_{e}$ is the angle between $v_{e}$ and $D$ momenta in the $e v_{e}$ rest frame and $\chi$ is the angle between the two decay planes. The dots with error bars are data, the blue curves are the weighted signal MC and the hatched histograms are the simulated backgrounds.

the ratios of the hadronic form factors to be $r_{V}=\frac{V(0)}{A_{1}(0)}=1.24 \pm 0.09_{\text {stat. }} \pm 0.06_{\text {sys. }}$ and $r_{2}=\frac{A_{2}(0)}{A_{1}(0)}=$ $1.05 \pm 0.15_{\text {stat. }} \pm 0.05_{\text {sys. }}$. Also, we search for $D^{+} \rightarrow \phi e^{+} v_{e}$, but do not find obvious signal. So, we set the upper limit on the branching fraction for $D^{+} \rightarrow \phi e^{+} v_{e}$ to be $1.3 \times 10^{-5}$ at $90 \%$ Confidence Level, which is significantly better than previous searches.

\section{3. $D$ hadronic decays}

We perform Dalitz plot analysis on the 3-body decay $D^{+} \rightarrow K_{S}^{0} \pi^{+} \pi^{0}$ [16]. Based on 166694 candidate events with a background of about $15 \%$, we fit the distribution of data to a coherent sum of six intermediate resonances plus a nonresonant component with a low mass scalar resonance $\bar{\kappa}$ included. From the analysis, we obtain the partial branching fractions for each component combing with the fitted fractions and the world averaged branching fraction for $D^{+} \rightarrow K_{S}^{0} \pi^{+} \pi^{0}(6.99 \pm$ $0.27) \%$ [17].

We determine the $D^{0} \bar{D}^{0}$ mixing parameter $y_{\mathrm{CP}}=\left(-2.1 \pm 1.3_{\text {stat. }} \pm 0.7_{\text {sys. }}\right) \%$, by analysis of $D^{0} \rightarrow K^{-} \ell^{+} v_{\ell}(\ell=e$ and $\mu)$ using the $\mathrm{CP}$ even tags $K^{+} K^{-}, \pi^{+} \pi^{-}$and $K_{S}^{0} \pi^{0} \pi^{0}$, and the CP odd tags $K_{S}^{0} \pi^{0}, K_{S}^{0} \eta$ and $K_{S}^{0} \omega$ [18]. This result is compatible with the previous measurement with about two standard deviations. However, the precision is still statistically limited and less precise than the current world average.

Measurement of the strong phase difference between $D^{0}$ and $\bar{D}^{0}$ is important to relate to the $D^{0} \bar{D}^{0}$ mixing parameters $x$ and $y$ from $x^{\prime}$ and $y^{\prime}$. We measure the $D \rightarrow K^{-} \pi^{+}$strong phase difference 

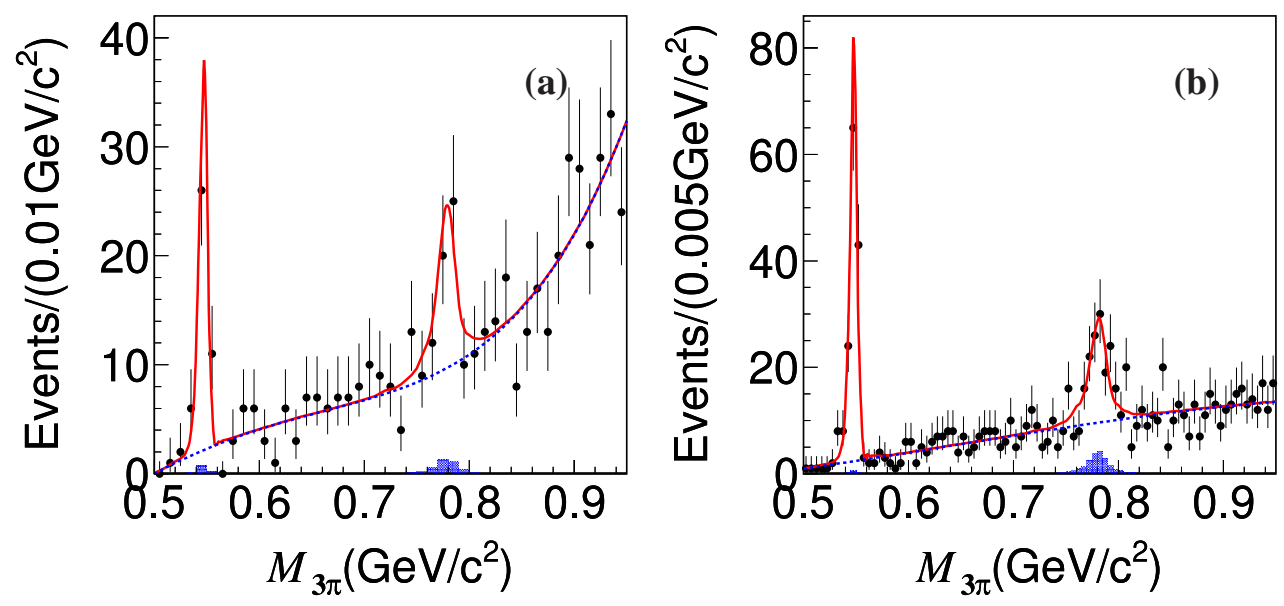

Figure 4: Fits to the $\pi^{+} \pi^{-} \pi^{0}$ invariant mass spectra of the selected (a) $D^{0} \rightarrow \omega \pi^{0}$ and (b) $D^{+} \rightarrow \omega \pi^{+}$. The blue hatched hitograms are the sideband background events.

based on analysis of $D^{0} \rightarrow K^{-} \pi^{+}$and $K^{+} \pi^{-}$using the CP even tags $K^{+} K^{-}, \pi^{+} \pi^{-}, K_{S}^{0} \pi^{0} \pi^{0}, \pi^{0} \pi^{0}$ and $\rho^{0} \pi^{0}$, and the CP odd tags $K_{S}^{0} \pi^{0}, K_{S}^{0} \eta$ and $K_{S}^{0} \omega[19]$. We determine the asymmetry of $\mathscr{A}_{K \pi}^{\mathrm{CP}}$ of the branching fraction of $D \rightarrow K^{-} \pi^{+}$in CP-odd and CP-even eignensates to be $(12.7 \pm 1.3 \pm 0.7) \%$. With external inputs of $r^{2}=(3.50 \pm 0.04) \times 10^{-3}, y=(6.7 \pm 0.9) \times 10^{-3}$ from HFAG [20] and $R_{W S}=(3.80 \pm 0.05) \times 10^{-3}$ from PDG [17]. The $\cos \delta_{K \pi}$ is determined to be $1.02 \pm 0.11_{\text {stat. }} \pm$ $0.06_{\text {sys. }} \pm 0.01_{\text {input }}$.

It is expected that $\mathscr{B}\left(D^{0(+)} \rightarrow \omega \pi^{0(+)}\right)$ is at $10^{-4}$ level [21]. CLEO searched for and did not observe the $D^{0} \rightarrow \omega \pi^{0}$ and $D^{+} \rightarrow \omega \pi^{+}$signals using single tag method [22]. They set the upper limits on these two decay branching fractions to be $2.6 \times 10^{-4}$ and $3.4 \times 10^{-4}$ at $90 \%$ confidence level, respectively. We search for $D^{0} \rightarrow \omega \pi^{0}$ and $D^{+} \rightarrow \omega \pi^{+}$by using double tag method [23] with the fitted $\pi^{+} \pi^{-} \pi^{0}$ invariant mass spectra shown in Fig. 4. The significance of the $D^{0} \rightarrow \omega \pi^{0}$ and $D^{+} \rightarrow \omega \pi^{+}$signals are $4.1 \sigma$ and $5.4 \sigma$, respectively. These two branching fractions are determined to be $\mathscr{B}\left(D^{0} \rightarrow \omega \pi^{0}\right)=\left(1.05 \pm 0.41_{\text {stat. }} \pm 0.09_{\text {sys. }}\right) \times 10^{-4}$ and $\mathscr{B}\left(D^{+} \rightarrow \omega \pi^{+}\right)=\left(2.74 \pm 0.58_{\text {stat. }} \pm\right.$ $\left.0.17_{\text {sys. }}\right) \times 10^{-4}$. Also, we confirm that the $\omega$ helicity angle of the $D^{0(+)} \rightarrow \omega \pi^{0(+)}$ candidates follow the expected $H_{\omega}^{2}=\cos ^{2} \theta_{\text {helicity }}$ formalism.

\section{4. $D$ rare decays}

Search for the FCNC and LNV rare decays of charmed mesons can shed some lights on new physics beyond the SM. At BESIII, we have searched for the rare decays of $D^{0} \rightarrow \gamma \gamma$ [24] and $D^{+} \rightarrow K(\pi)^{ \pm} e^{\mp} e^{+}[25]$ with double and single tag methods, respectively. No significant signals are observed, thus we set the upper limits of their branching fractions to be $\mathscr{B}\left(D^{0} \rightarrow \gamma \gamma\right)<3.8 \times 10^{-6}$, $\mathscr{B}\left(D^{+} \rightarrow K^{+} e^{+} e^{-}\right)<1.2 \times 10^{-6}, \mathscr{B}\left(D^{+} \rightarrow K^{-} e^{+} e^{+}\right)<0.6 \times 10^{-6}, \mathscr{B}\left(D^{+} \rightarrow K^{+} e^{+} e^{-}\right)<0.3 \times$ $10^{-6}, \mathscr{B}\left(D^{+} \rightarrow K^{-} e^{+} e^{+}\right)<1.2 \times 10^{-6}$ at $90 \%$ confidence level. Some of them are improved compared to previous measurements. 

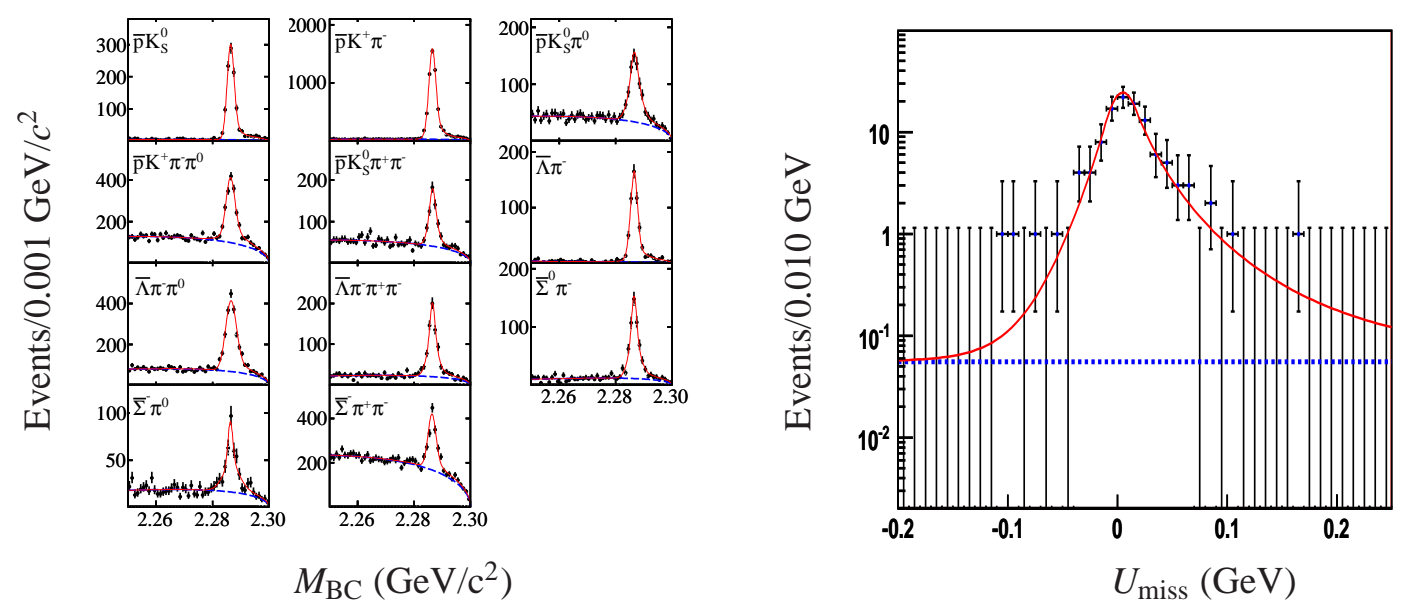

Figure 5: The distribution of (left) fits to the $M_{\mathrm{BC}}$ distributions for different single tag modes and (right) fit to the $U_{\text {miss }}$ distribution within the $\Lambda$ signal region.

\section{5. $\Lambda_{c}^{+}$semileptonic decay}

The $\Lambda_{c}^{+}$was observed in $e^{+} e^{-}$annihilation at Mark II in 1979 [26]. Thereafter, many works have been done to study the $\Lambda_{c}^{+}$decay properties. However, the knowledge of $\Lambda_{c}^{+}$physics are still very poor [1]. The sum of the branching fractions of the known $\Lambda_{c}^{+}$decays is not more than $65 \%$ and their uncertainties are quite large. So, significantly improved measurements of these decay branching fractions are important to comprehensively understand the $\Lambda_{c}^{+}$decay properties. we performed the the first absolute measurement of $\mathscr{B}\left(\Lambda_{c}^{+} \rightarrow \Lambda e^{+} v_{e}\right)$ by analyzing $567 \mathrm{pb}^{-1}$ [2] of data accumulated at $\sqrt{s}=4.599 \mathrm{GeV}$ with the BESIII detector at the BEPCII collider [27]. This is the largest $\Lambda_{c}^{+}$data sample near the $\Lambda_{c}^{+} \bar{\Lambda}_{c}^{-}$threshold, where the $\Lambda_{c}^{+}$is always produced in association with a $\bar{\Lambda}_{c}^{-}$baryon. Hence, $\mathscr{B}\left(\Lambda_{c}^{+} \rightarrow \Lambda e^{+} v_{e}\right)$ can be accessed by measuring the relative probability of finding the semileptonic decay when the $\bar{\Lambda}_{c}^{-}$is reconstructed in a number of prolific decay channels. This will provide a clean and straightforward BF measurement without requiring knowledge of the total number of $\Lambda_{c}^{+} \bar{\Lambda}_{c}^{-}$events produced.

The $\bar{\Lambda}_{c}^{-}$are reconstructed using eleven singly hadronic decay modes: $\bar{\Lambda}_{c}^{-} \rightarrow \bar{p} K_{S}^{0}, \bar{p} K^{+} \pi^{-}$, $\bar{p} K_{S}^{0} \pi^{0}, \bar{p} K^{+} \pi^{-} \pi^{0}, \bar{p} K_{S}^{0} \pi^{+} \pi^{-}, \bar{\Lambda} \pi^{-}, \bar{\Lambda} \pi^{-} \pi^{0}, \bar{\Lambda} \pi^{-} \pi^{+} \pi^{-}, \bar{\Sigma}^{0} \pi^{-}, \bar{\Sigma}^{-} \pi^{0}$ and $\bar{\Sigma}^{-} \pi^{+} \pi^{-}$, where the intermediate particles $K_{S}^{0}, \bar{\Lambda}, \bar{\Sigma}^{0}, \bar{\Sigma}^{-}$and $\pi^{0}$ are reconstructed by their decays into $K_{S}^{0} \rightarrow \pi^{+} \pi^{-}$, $\bar{\Lambda} \rightarrow \bar{p} \pi^{+}, \bar{\Sigma}^{0} \rightarrow \gamma \bar{\Lambda}$ with $\bar{\Lambda} \rightarrow \bar{p} \pi^{+}, \bar{\Sigma}^{-} \rightarrow \bar{p} \pi^{0}$ and $\pi^{0} \rightarrow \gamma \gamma$, respectively. The single tagged $\bar{\Lambda}_{c}^{-}$ signals are identified using the beam constrained mass, $M_{\mathrm{BC}}=\sqrt{E_{\text {beam }}^{2}-\left|\vec{p}_{\bar{\Lambda}_{c}^{-}}\right|^{2}}$, where $E_{\text {beam }}$ is the beam energy and $\vec{p}_{\bar{\Lambda}_{c}^{-}}$is the momentum of the $\bar{\Lambda}_{c}^{-}$candidate. The $M_{\mathrm{BC}}$ distributions are shown in Fig. 5 (left). Finally, we obtain the total single tag yield summed over all 11 modes to be $N_{\bar{\Lambda}_{c}^{-}}^{\text {tot }}=$ $14415 \pm 159$. Candidate events for $\Lambda_{c}^{+} \rightarrow \Lambda e^{+} v_{e}$ are selected from the remaining tracks recoiling against the single tag $\bar{\Lambda}_{c}^{-}$candidates. After subtracting the background, we obtain $103.5 \pm 10.9$ net signal yields for $\Lambda_{c}^{+} \rightarrow \Lambda e^{+} v_{e}$ and measured the branching fraction $\mathscr{B}\left(\Lambda_{c}^{+} \rightarrow \Lambda e^{+} v_{e}\right)=(3.63 \pm$ $\left.0.38_{\text {stat. }} \pm 0.20_{\text {sys. }}\right) \%$, which provide the first direct measurement of the absolute branching fraction for $\Lambda_{c}^{+} \rightarrow \Lambda e^{+} v_{e}$ and provide a stringent test on non-perturbative models. 

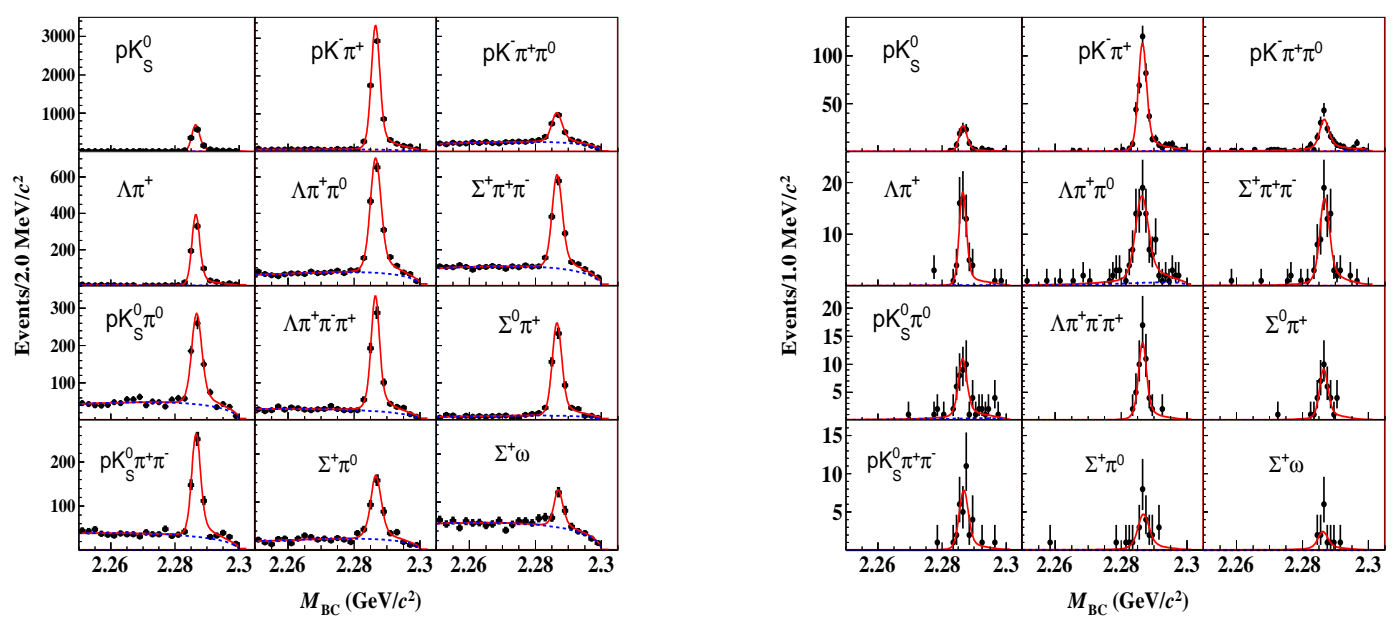

Figure 6: Fits to (left) $M_{\mathrm{BC}}$ distributions for 12 single tag modes and (right) $M_{\mathrm{BC}}$ distributions for the 12 double tag modes.

\section{6. $\Lambda_{c}^{+}$hadronic decay}

We also study 12 hadronic decays of $\Lambda_{c}^{+}$, which are $\Lambda_{c}^{+} \rightarrow p K_{S}^{0}, p K^{-} \pi^{+}, p K_{S}^{0} \pi^{0}, p K_{S}^{0} \pi^{+} \pi^{-}$, $\Lambda \pi^{+}, \Lambda \pi^{+} \pi^{0}, \Lambda \pi^{+} \pi^{+} \pi^{-}, p K^{-} \pi^{+} \pi^{0}, \Sigma^{0} \pi^{+}, \Sigma^{+} \pi^{0}, \Sigma^{+} \pi^{+} \pi^{-}$and $\Sigma^{+} \omega$ [28]. The $M_{\mathrm{BC}}$ distributions for the single tags and the double tags are shown in Fig. 6 (left) and Fig. 6 (right), respectively. Then we used a least-squares fitter, combing the yields from both single tags and double tags, to obtain these branching fractions of the $12 \Lambda_{c}^{+}$hadronic decay modes globally. We measured $\mathscr{B}\left(\Lambda_{c}^{+} \rightarrow\right.$ $\left.p K_{S}^{0}\right)=(1.52 \pm 0.08 \pm 0.03) \%, \mathscr{B}\left(\Lambda_{c}^{+} \rightarrow p K^{-} \pi^{+}\right)=(5.84 \pm 0.27 \pm 0.23) \%, \mathscr{B}\left(\Lambda_{c}^{+} \rightarrow p K_{S}^{0} \pi^{0}\right)=$ $(5.84 \pm 0.27 \pm 0.23) \%, \mathscr{B}\left(\Lambda_{c}^{+} \rightarrow p K_{S}^{0} \pi^{+} \pi^{-}\right)=(1.53 \pm 0.11 \pm 0.09) \%, \mathscr{B}\left(\Lambda_{c}^{+} \rightarrow p K^{-} \pi^{+} \pi^{0}\right)=$ $(4.53 \pm 0.23 \pm 0.30) \%, \mathscr{B}\left(\Lambda_{c}^{+} \rightarrow \Lambda \pi^{+}\right)=(1.24 \pm 0.07 \pm 0.03) \%, \mathscr{B}\left(\Lambda_{c}^{+} \rightarrow \Lambda \pi^{+} \pi^{0}\right)=(7.01 \pm$ $0.37 \pm 0.19) \%, \mathscr{B}\left(\Lambda_{c}^{+} \rightarrow \Lambda \pi^{+} \pi^{+} \pi^{-}\right)=(3.81 \pm 0.24 \pm 0.18) \%, \mathscr{B}\left(\Lambda_{c}^{+} \rightarrow \Sigma^{0} \pi^{+}\right)=(1.27 \pm 0.08 \pm$ $0.03) \%, \mathscr{B}\left(\Lambda_{c}^{+} \rightarrow \Sigma^{+} \pi^{0}\right)=(1.18 \pm 0.10 \pm 0.03) \%, \mathscr{B}\left(\Lambda_{c}^{+} \rightarrow \Sigma^{+} \pi^{+} \pi^{-}\right)=(4.25 \pm 0.24 \pm 0.20) \%$ and $\mathscr{B}\left(\Lambda_{c}^{+} \rightarrow \Sigma^{+} \omega\right)=(1.56 \pm 0.20 \pm 0.07) \%$, where the uncertainties are statistical and the second systematic. These results are more precise than the PDG values [1]. The $\mathscr{B}\left(\Lambda_{c}^{+} \rightarrow p K^{-} \pi^{+}\right)$ measured in this wok and the one measured at BELLE [29] will calibrate other decay rates of $\Lambda_{c}^{+}$ with much better precisions.

We also studied the first direct measurement of the $\Lambda_{c}^{+}$decays involving the neutron with the double tag method [30]. We performd a two-dimensions unbinned likelihood fit to the $M_{\text {miss }}^{2}$ and $M_{\pi^{+} \pi^{-}}$distributions in both $M_{\mathrm{BC}}$ signal and sideband regions simultaneously. The fitting is shown in Fig. 7. We modelled the $M_{\pi^{+} \pi^{-}}$and $M_{\text {miss }}^{2}$ distributions with a product of two onedimensional probability density functions (PDF), one for each dimension. The signal functions for $M_{\text {miss }}^{2}$ and $M_{\pi^{+} \pi^{-}}$are both described by a double Gaussian function. The peaking background in $M_{\text {miss }}^{2}$ distribution is described by a double Gaussian function with parameters fixed according to MC simulations, and the flat distribution in the $M_{\pi^{+} \pi^{-}}$spectrum is described by a constant function. The non- $\Lambda_{c}^{+}$decay background is modelled by a second-order polynomial function in the $M_{\text {miss }}^{2}$ distribution and a Gaussian function plus a second-order polynomial function in the $M_{\pi^{+} \pi^{-}}$ 

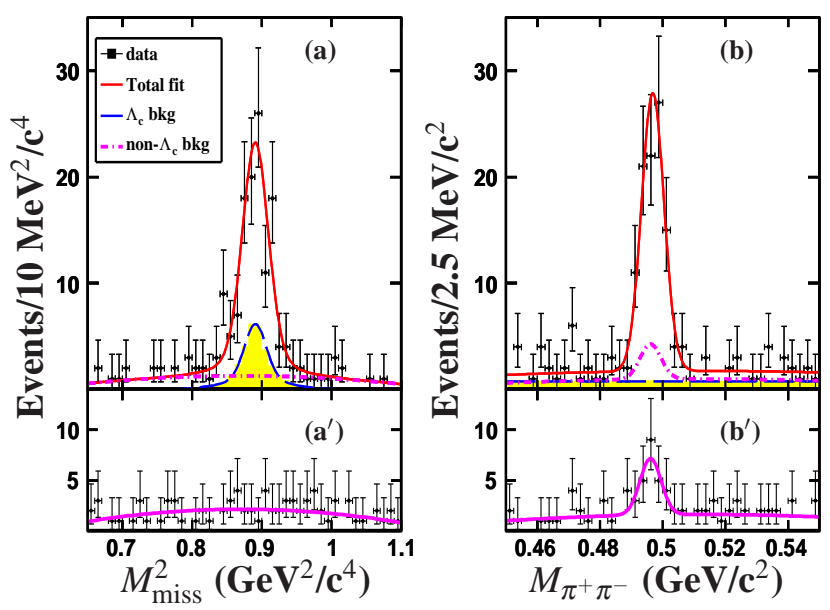

Figure 7: Simultaneous fit to $M_{\text {miss }}^{2}$ and $M_{\pi^{+} \pi^{-}}$of events in $(\mathrm{a}, \mathrm{b})$ the $\bar{\Lambda}_{c}^{-}$signal region and $\left(\mathrm{a}^{\prime}, \mathrm{b}^{\prime}\right)$ sideband regions. Data are shown as the dots with error bars. The long-dashed lines (blue) show the $\Lambda_{c}^{+}$backgrounds while the dot-dashed curves (pink) show the non- $\Lambda_{c}^{+}$backgrounds. The red curves show the total fit. The (yellow) shaded area show the MC simulated backgrounds from $\Lambda_{c}^{+}$decay.

distribution, in which the parameters and the normalized background numbers are constrained by the events in $M_{\mathrm{BC}}$ sideband in the simultaneous fit. We obtain $83.2 \pm 10.6$ signal events for $\Lambda_{c}^{+} \rightarrow$ $n K_{S}^{0} \pi^{+}$and measured the absolute branching fraction to be $\mathscr{B}\left(\Lambda_{c}^{+} \rightarrow n K_{S}^{0} \pi^{+}\right)=(1.82 \pm 0.23 \pm$ $0.11) \%$. This is the first direct measurement of $\Lambda_{c}^{+}$decays involving the neutron in final states experimentally, since $\Lambda_{c}^{+}$has been discovered more than 30 years ago. According to the measured $\mathscr{B}\left(\Lambda_{c}^{+} \rightarrow p K^{-} \pi^{+}\right)$and $\mathscr{B}\left(\Lambda_{c}^{+} \rightarrow p K_{S}^{0} \pi^{0}\right)$ at BESIII [28], we have $\mathscr{B}\left(\Lambda_{c}^{+} \rightarrow n \bar{K}^{0} \pi^{+}\right) / \mathscr{B}\left(\Lambda_{c}^{+} \rightarrow\right.$ $\left.p K^{-} \pi^{+}\right)=0.62 \pm 0.09$ and $\left.\mathscr{B}\left(\Lambda_{c}^{+} \rightarrow n \bar{K}^{0} \pi^{+}\right) / \mathscr{B}\left(\Lambda_{c}^{+} \rightarrow p \bar{K}^{0} \pi^{0}\right)\right)=0.97 \pm 0.16$, in which the common uncertainties have been cancelled in the calculation. These ratios are useful to test the isospin symmetry and extract strong phases of different final states [31]. The measurement of the neutron mode in this work provides the first complementary data to the previously measured proton-involved decays, which is a significant progress in studying the $\Lambda_{c}^{+}$.

\section{Summary}

In conclusion, by analyzing 2.93 and $0.567 \mathrm{fb}^{-1}$ data taken at $\sqrt{s}=3.773$ and $4.599 \mathrm{GeV}$ with the BESIII detector, we report the precise measurements of the decay constant $f_{D^{+}}$, the form factors of $D$ semileptonic decays, the Dalitz plot analysis of $D^{+} \rightarrow K_{S}^{0} \pi^{+} \pi^{0}$, the strong phase measurement in $D^{0} \rightarrow K^{-} \pi^{+}$, the $D^{0} \bar{D}^{0}$ mixing parameter $y_{\mathrm{CP}}$, the observation of the singly-cabibbo-suppressed hadronic decays $D^{+} \rightarrow \omega \pi^{+}$and $D^{0} \rightarrow \omega \pi^{0}$, as well as the first absolute measurements of the branching fractions for $\Lambda_{c}^{+} \rightarrow \Lambda e^{+} v_{e}, \Lambda_{c}^{+}$decays to 12 hadronic final states and the first direct measurement of $\Lambda_{c}^{+}$decays involving the neutron $\Lambda_{c}^{+} \rightarrow n K_{S}^{0} \pi^{+}$. These are important to test the LQCD calculations on $f_{D^{+}}$and the form factors of $D$ semileptonic decays, to test the CKM matrix unitarity, to search for new physics beyond the SM, and to comprehensively understand the $\Lambda_{c}^{+}$ decay property. More interesting physics based on charmed mesons and charmed baryons are hopefully achieved at BESIII in the near future. 


\section{References}

[1] K. A. Olive, et al. (Particle Data Group), Chin. Phys. C 38, 090001 (2014).

[2] M. Ablikim, et al. (BESIII Collaboration), Chin. Phys. C 37, 123001 (2013).

[3] M. Ablikim, et al. (BESIII Collaboration), Chin. Phys. C 39, 093001 (2015).

[4] M. Ablikim, et al. (BESIII Collaboration), Nucl. Phys. Meth. A 614, 345 (2010).

[5] M. Ablikim, et al. (BESIII Collaboration), Phys. Rev. D 89, 051104(R) (2014).

[6] E. Follana, et al. (HPQCD and UKQCD Collaborations), Phys. Rev. Lett. 100, 062002 (2008).

[7] M. Ablikim, et al. (BESIII Collaboration), Phy. Rev. D 92 (2015) 072012.

[8] D. Becirevic and A. B. Kaidalov, Phys. Lett. B 478, 417 (2000).

[9] T. Becher, R. J. Hill, Phys. Lett. B 633, 61 (2006).

[10] H. Na, et al. (HPQCD Collaboration), Phys. Rev. D 82, 114506 (2010).

[11] H. Na, et al. (HPQCD Collaboration), Phys. Rev. D 84, 114505 (2010).

[12] M. Ablikim, et al. (BESIII Collaboration), Phys. Rev. D 92, 112008 (2015).

[13] M. Ablikim, et al. (BESIII Collaboration), arXiv:1512.08627[hep-ex].

[14] J. G. Korner and G. A. Schuler, Z. Phys. C 46, 93 (1990); F. J. Gilman and R. L. Singleton, Jr., Phys. Rev. D 41, 142 (1990); J. M. Link, et al. (FOCUS Collaboration), Phys. Lett. B 54 544, 89 (2002).

[15] M. Ablikim, et al. (BESIII Collaboration), Phy. Rev. D 92 (2015) 071101.

[16] M. Ablikim, et al. (BESIII Collaboration), Phys. Rev. D 89, 052001 (2014).

[17] J. Beringer, et al. (Particle Data Group), Phys. Rev. D 86, 010001 (2012).

[18] M. Ablikim, et al. (BESIII Collaboration), Phys. Lett. B 744, 339 (2015).

[19] M. Ablikim, et al. (BESIII Collaboration), Phys. Lett. B 734, 227 (2014).

[20] Heavy Flavor Averaging Group, http://www.slac.stanford.edu/xorg/hfag/charm/.

[21] Hai-Yang Cheng and Cheng-Wei Chiang, Phys. Rev. D 81, 074021 (2010).

[22] P. Rubin, et al. (CLEO Collaboration), Phys. Rev. Lett. 96, 081802 (2006).

[23] M. Ablikim, et al. (BESIII Collaboration), Phy. Rev. Lett. 116 (2016) 082001.

[24] M. Ablikim, et al. (BESIII Collaboration), Phy. Rev. D 91 (2015) 112015.

[25] Minggang Zhao (For BESIII Collaboration), talk presented at Charm2015.

[26] G. S. Abrams, et al. [Mark II Collaboration], Phys. Rev. Lett. 44, 10 (1980).

[27] M. Ablikim, et al. (BESIII Collaboration), Phy. Rev. Lett. 115 (2015) 221805.

[28] M. Ablikim, et al. (BESIII Collaboration), Phy. Rev. Lett. 116 (2016) 052001.

[29] A. Zupanc, et al. (Belle Collaboration), Phys. Rev. Lett. 58 113, 042002 (2014).

[30] Lei Li (For BESIII Collaboration), talk presented at Bormio 2016.

[31] Cai-Dian Lü, Wei Wang and Fu-Sheng Yu, Phys. Rev. D 93, 056008 (2016); H. Y. Cheng, Front. Phys. 10(6), 101406 (2015). 